\title{
Vergleich der Exposition der Bevölkerung durch digitale und analoge Rundfunksender
}

\author{
M. Schubert ${ }^{1}$, C. Bornkessel ${ }^{1}$, M. Wuschek ${ }^{2}$, and P. Schmidt ${ }^{3}$ \\ ${ }^{1}$ IMST GmbH, Kamp-Lintfort, Deutschland \\ ${ }^{2}$ Fachhochschule Deggendorf, Deggendorf, Deutschland \\ ${ }^{3}$ EM-Institut GmbH, Regensburg, Deutschland
}

\begin{abstract}
Zusammenfassung. Dieser Beitrag fasst die Ergebnisse eines Projektes zusammen, das vom Bundesamt für Strahlenschutz und dem Ministerium für Umwelt, Naturschutz und Reaktorsicherheit (BMU) im Rahmen des Deutschen Mobilfunk Forschungsprogramms (DMF) gefördert wurde. Hintergrund der Studie ist die Untersuchung der Auswirkung der Umstellung von der analogen auf die digitale terrestrische Rundfunkübertragung in Deutschland auf die Exposition der Bevölkerung.
\end{abstract}

\section{Einleitung}

DAB (Digital Audio Broadcast) und DVB-T (Digital Video Broadcast Terrestrial) sollen als neue digitale Technologien mittelfristig die bestehende analoge terrestrische Radio- und Fernsehübertragung ersetzen. Welche Auswirkungen dies in Bezug auf die Exposition der Bevölkerung durch hochfrequente elektromagnetische Felder haben wird, ist derzeit weitgehend ungeklärt.

Die Umstellung auf DVB-T wird in Deutschland nicht landesweit durchgeführt, sondern als erster Schritt in Form von sogenannten Startinseln, die sich zunächst auf dicht besiedelte Regionen beschränken. Je nach Empfangssituation können drei Versorgungsklassen unterschieden werden. In der ,portable indoor"-Region ist ein Empfang der digitalen Signale mit Hilfe einer kleinen Zimmerantenne, in der ,,portable outdoor"-Region mittels einer kleinen Stabantenne und in der ,fixed antenna“-Region mit einer Richtantenne auf dem Gebäudedach möglich (Cept, 1997).

Nachdem geeignete Messverfahren zur Erfassung der Exposition durch die unterschiedlichen Funkdienste entwickelt wurden, wurde eine Messreihe durchgeführt, um die Expositionen von DAB und DVB-T mit ihren analogen Pendants

Correspondence to: $\mathrm{M}$. Schubert

(schubert@imst.de) zu vergleichen. Darüber hinaus wurde ein einfaches Berechnungsverfahren entwickelt, mit dem die messtechnisch erhobenen Immissionen auch mit entsprechenden Berechnungen verglichen wurden.

\section{Verfahren}

\subsection{Messverfahren}

Die spektralen Messungen erfolgen mit einem Spektrumanalysator und einer geeigneten Empfangsantenne. Zur (frequenzselektiven) Messung der Felder von Ton- und Fernsehrundfunksendern sind einige wichtige Einstellparameter am Spektrumanalysator korrekt zu wählen (insbesondere eine geeignete Auflösebandbreite RBW und der richtige Detektortyp), um die in den einschlägigen Normen geforderte RMS-Erfassung sicherzustellen.

Für die Erfassung von frequenzmodulierten Signalen des UKW-Rundfunks ist der RMS-Detektor nicht notwendig. Die Auflösebandbreite (RBW) sollte zwischen 100 und $300 \mathrm{kHz}$ liegen.

Bei analogen TV-Signalen erfolgt die Ermittlung der mittleren Immission durch Messung der Synchronspitzenleistung mit dem Peak-Detektor mit anschließender Verringerung des Messergebnisses um einen Korrekturfaktors von etwa $4 \mathrm{~dB}$ zur Berücksichtigung eines mittleren Bildinhalts unter Einschluss der Tonsignale.

Bei $D A B$ und DVB-T Signalen sind die Messungen unbedingt mit dem RMS-Detektor durchzuführen. Je nach verwendetem Messgerät ist auf eine ausreichend große Messzeit zu achten, da der RMS-Detektor bei zu kleinen Messzeiten zu hohe Messergebnisse liefert. Eine an die Signalbandbreite angepasste RBW ist einzustellen.

Die Messunsicherheit unter Berücksichtigung von messeinrichtungsbedingten und methodenbedingten Faktoren liegt im Bereich von $3 \mathrm{~dB}$ (Vertrauensintervall 


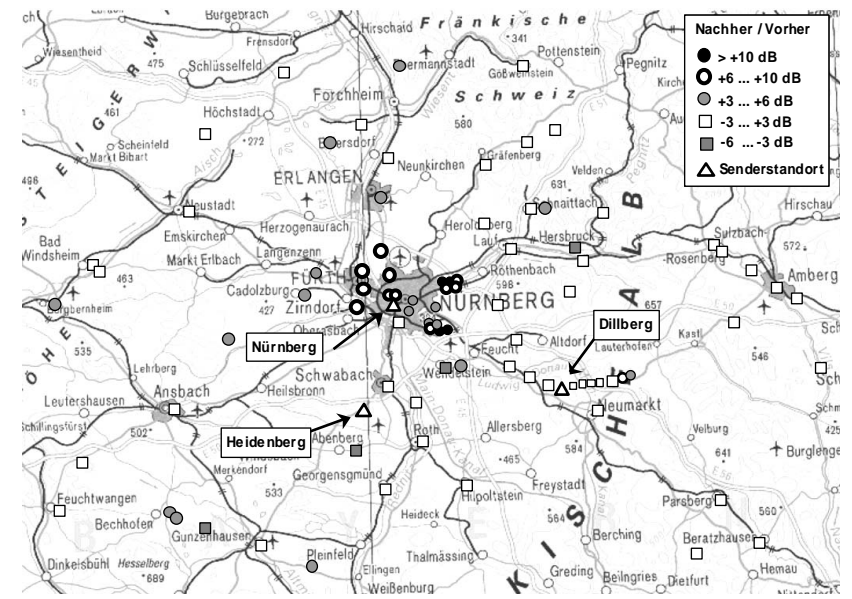

Abb. 1. Quotient der Leistungsflussdichtewerte (Gesamtimmission Ton- und Fernsehrundfunk) aus „Nach-“ und „Vorhermessung“ in $\mathrm{dB}$ für die 87 Messpunkte im Raum Nürnberg (Darstellung in fünf Klassen).

95\%) und damit im üblichen Bereich für HochfrequenzImmissionsmessungen.

\subsection{Berechnungsverfahren}

Als Grundlage für das selbst entwickelte Programm FPT diente die Empfehlung ITU-R P.1546 der International Telecommunications Union. Diese stellt eine allgemeine Vorhersagemethode für elektrische Feldstärken in Form von Ausbreitungskurven dar, die durch eine statistische Auswertung einer großen Anzahl von Messungen ermittelt wurden. Diese Methode wurde an die hier vorliegende Problematik angepasst und darüber hinaus noch in der Art erweitert, dass auch Aussagen über die Immissionen in einer Entfernung unterhalb von $1 \mathrm{~km}$ vom Sender möglich sind. Sie zeichnet sich durch eine einfache Handhabung und einen geringen Rechenzeitbedarf aus.

\section{Ergebnisse}

\subsection{Expositionserhebung}

Im Rahmen der Expositionserhebung wurden Messungen an mehr als 300 Punkten in den DVB-T-Startregionen München und Nürnberg durchgeführt. Für 200 statistisch unabhängig verteilte Messpunkte (Bernkopf 2004) wurden umfangreiche „Vorher-Nachher"-Untersuchungen (d.h. vor und nach der Umstellung DVB-T) vorgenommen, um eine mögliche Expositionsveränderung durch die Einführung von DVB-T zu erkennen.

Die gefundenen Expositionswerte zeigen eine große Spannweite bezüglich ihrer absoluten Höhe von mehr als $50 \mathrm{~dB}$. Bezüglich der Grenzwertausschöpfung ergab sich

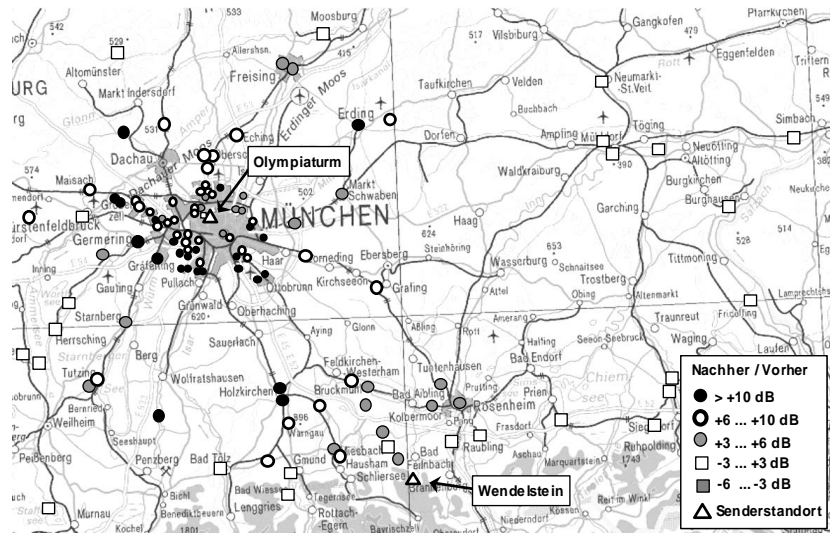

Abb. 2. Quotient der Leistungsflussdichtewerte (Gesamtimmission Ton- und Fernsehrundfunk) aus „Nach-“ und „Vorhermessung“" in dB für die 113 Messpunkte im Raum München (Darstellung in fünf Klassen).

am Punkt mit den höchsten Summenimmissionen ein leistungsflussdichtebezogener Ausschöpfungsgrad von nur etwa 3 Promille.

Ein Vergleich der Ergebnisse der „Vorher-“ bzw. „Nachhermessungen“" an den Messpunkten in der Region Nürnberg zeigt eine deutliche Zunahme der Gesamtexposition im Bereich der Stadtgebiete Nürnberg / Fürth / Erlangen (mittlere Zunahme: $6,8 \mathrm{~dB}$ ). Grund dafür ist die Verlagerung der TV-Sender im Rahmen der Umstellung auf DVB-T vom Heidenberg südlich Schwabach zum Fernmeldeturm Nürnberg. Im Bereich „Dillberg” ist nur eine moderate Expositionszunahme von 1,5 dB feststellbar. Die Messpunkte außerhalb der Versorgungsklasse ,,portable indoor” zeigen keine signifikante Veränderung der mittleren Gesamtexposition (siehe Abb. 1).

Ein Vergleich der Ergebnisse der „Vorher-“ bzw. „Nachhermessungen " an den Messpunkten in der Region München zeigt eine signifikante Immissionszunahme im Stadtgebiet von München und im Bereich der direkt benachbarten Landkreise bis hin zum Wendelstein (mittlere Zunahme: 6,5 dB). Grund dafür sind einerseits die Zunahme der ERP am Wendelstein und am Olympiaturm in München durch die Einführung von DVB-T als auch eine veränderte Montagehöhe der Antennen am Olympiaturm in München im Vergleich zur „Vorhermessung“. In größerer Entfernung zum Olympiaturm in München bzw. Wendelstein ist keine signifikante Veränderung der Exposition mehr feststellbar (siehe Abb. 2).

Die Veränderung der ERP an dem für die betrachtete Region jeweils verantwortlichen Senderstandort kann als grober Indikator für die mittlere Expositionsänderung herangezogen werden. In der Region „Dillberg“ stimmen ERP-Zunahme und mittlere Expositionsänderung sehr gut überein.

Will man die hier gefundenen Ergebnisse auf andere Regionen in Deutschland übertragen, gilt das oben gesagte 
Tabelle 1. Mittlere Abweichung der berechneten Immissionen verglichen mit den entsprechenden Messwerten.

\begin{tabular}{llll}
\hline System & Korrektur & $\begin{array}{l}\text { Anzahl der } \\
\text { Punkte }\end{array}$ & $\begin{array}{l}\text { Mittlere Abweichung (dB) } \\
\text { (mit Zuschlägen) }\end{array}$ \\
\hline Analog TV & ja & 1.135 & 9,0 \\
DVB-T & ja & 948 & 11,5 \\
UKW & ja & 2.804 & 8,3 \\
DAB (landesweit) & ja & 181 & 7,3 \\
DAB (regional) & ja & 112 & 7,8 \\
\hline
\end{tabular}

analog: Nur in den ,portable indoor" versorgten Regionen der aktuell in Deutschland vorhandenen DVB-T-Inseln ist derzeit eine belastbare Aussage über die mittlere Expositionsänderung möglich, da nur dort der Wechsel von analoger auf digitale Versorgung nahezu vollständig stattgefunden hat. Ein ERP-Vergleich der für die Versorgung verantwortlichen Standorte kann in erster Näherung Aufschluss über eine gegebenenfalls vorhandene Expositionsänderung geben. ERP-Betrachtungen für zwei andere Regionen Deutschlands zeigen, dass, abhängig von der ERP der Digitalsender im Vergleich zu den abgeschalteten Analogsendern, sowohl eine Zunahme als auch eine Abnahme der mittleren Exposition durch die Einführung von DVB-T in der Umgebung von Senderstandorten möglich ist. Zusätzlich sollte jedoch nie vergessen werden, dass noch andere Faktoren als die ERP einen signifikanten Einfluss auf die mittlere Exposition ausüben können (z.B. eine veränderte Montagehöhe der Sendeantennen).

Ein Vergleich zwischen UKW- und DAB-Signalen ergibt eine deutliche Dominanz der UKW-Signale (Unterschiedsfaktor im Mittel größer als $10 \mathrm{~dB}$ ). Zukünftig vorgesehene Leistungserhöhungen bei DAB zur Gewährleistung einer zuverlässigeren Indoorversorgung werden diese derzeitige Differenz allerdings verkleinern.

\subsection{Vergleich zwischen Berechnung und Messung}

Für den Vergleich zwischen den gemessenen Immissionswerten vom analogen Fernsehen, DVB-T, UKW und DAB und den entsprechend rechentechnisch mit FPT prognostizierten Werten standen insgesamt 5180 Vergleichspaare zur Verfügung. Das Berechnungsprogramm wurde zunächst im Hinblick daraufhin betrachtet, dass es eine möglichst geringe mittlere betragsmäßige Abweichung bezüglich entsprechender Messwerte liefern sollte, d.h. der betragsmäßige Unterschied zwischen der rechentechnisch ermittelten und messtechnisch erfassten Immission soll möglichst gering sein, was als ein Maß für die Genauigkeit der rechentechnischen Prognose angesehen werden kann.

Die Berechnung mit Korrekturfaktor für eine von $10 \mathrm{~m}$ geänderte Empfangsantennenhöhe (laut ITU-Empfehlung) wurde anhand eines konfigurationsabhängigen Zuschlags im
Hinblick auf die mittlere Abweichung optimiert. Die einzelnen Konfigurationen und die zugehörigen Zuschläge für die Optimierung auf eine möglichst geringe mittlere Abweichung sind in Tabelle 1 aufgeteilt nach dem jeweiligem System dargestellt.

Die Tabelle 1 zeigt, dass die Prognose mit dem Programm FPT aufgrund der Größe der mittleren Abweichung nur eingeschränkt geeignet ist, die Immissionen durch Rundfunksender zuverlässig zu berechnen. Hierbei ist allerdings zu beachten, dass je nach betrachtetem Rundfunkdienst zwischen 78 und $83 \%$ aller Punkte eine maximale Abweichung von weniger als $\pm 3 \mathrm{~dB}$ zeigten. Für diese Punkte, die im gesamten jeweiligen Versorgungsgebiet verteilt lagen, liefert FPT trotz der wenigen Eingabeparameter eine vor dem Hintergrund der Messunsicherheit gute Immissionsprognose. Da es aber auch Punkte gibt, an denen zum Teil auch durch ihre geographische Lage begründet mit deutlichen Über- bzw. Unterschätzungen gerechnet werden muss, ist das Verfahren in der Praxis nur eingeschränkt geeignet, so dass letztlich auf Messungen nicht verzichtet werden kann.

Anschließend wurden die Berechnungsergebnisse mit einem erneuten Zuschlagsfaktor in der Art optimiert, dass möglichst alle rechentechnisch prognostizierten Werte oberhalb der gemessenen Immissionen lagen. Dieser Worst Case Ansatz kann für alle betrachteten Rundfunkdienste als geeignet angesehen werden. Hierdurch konnte demnach im gesamten Versorgungsgebiet eines Senders eine Immission prognostiziert werden, die messtechnisch vor dem Hintergrund der Messunsicherheit nicht unterschritten wurde. Hierbei ist aber zur berücksichtigen, dass die mittlere Abweichung bezüglich der Messergebnisse stark ansteigt und Werte von 20 bis $30 \mathrm{~dB}$ erreicht.

\section{Zusammenfassung}

Der Vergleich der Ergebnisse der „,Vorher-“ bzw. „Nachhermessungen" an den Messpunkten zweier ausgewählter Regionen zeigt eine Zunahme der mittleren Exposition. Gründe für die Erhöhung liegen zum einen in der Verlagerung der TV-Sender im Rahmen der Umstellung auf DVB-T und zum anderen an der Zunahme der ERP der Sendeanlagen nach der Einführung von DVB-T in den betrachteten Gebieten. 
Da dies aber nicht für alle Startinseln in Deutschland zutrifft, sind die hier gefundenen Ergebnisse nicht allgemein auf andere Startregionen übertragbar.

Ein Vergleich zwischen UKW- und DAB-Signalen ergibt eine deutliche Dominanz der UKW-Signale (Unterschiedsfaktor im Mittel größer als $10 \mathrm{~dB}$ ). Zukünftig vorgesehene Leistungserhöhungen bei DAB werden die derzeitige Differenz allerdings verkleinern.

Ein einfaches auf der ITU-R P.1546 basierendes Berechnungsprogramm wurde entwickelt und die messtechnisch erhobenen Immissionen auch mit entsprechenden Berechnungen verglichen. Eine Optimierung dieser Berechnungsergebnisse resultiert in einer mittleren Abweichung von 7,3 bis $11,5 \mathrm{~dB}$ je nach betrachtetem System. Vor dem Hintergrund einer Messunsicherheit von ca. $\pm 3 \mathrm{~dB}$ zeigt diese Methode eine gute Übereinstimmung zwischen Messung und Berechnung in 78-83\% aller Punkte. Eine andere Optimierung im Hinblick darauf, dass die rechentechnisch prognostizierte Immission an allen Punkten oberhalb der gemessenen Immission liegt, zeigt eine mittlere Abweichung von 20-30 dB.
Danksagung. Diese Studie wurde gefördert vom Bundesamt für Strahlenschutz und dem Ministerium für Umwelt, Naturschutz und Reaktorsicherheit (BMU) im Rahmen des Deutschen Mobilfunk Forschungsprogramms (DMF).

\section{Literatur}

Bernkopf J.: Monitoring elektromagnetischer Felder an statistisch ausgewählten Orten in Bayern, NIR 2004 Nichtionisierende Strahlung Sicherheit und Gesundheit, 36. Jahrestagung des Fachverbandes für Strahlenschutz, Köln, 411-418, 2004.

CEPT: The Chester 1997 multilateral coordination agreement relating to technical criteria, coordination principles and procedures for the introduction of terrestrial digital video broadcasting (DVB-T), Chester, 1997.

ITU-R P.1546-1: Method for point-to-area predictions for terrestrial services in the frequency range $30 \mathrm{MHz}$ to $3000 \mathrm{MHz}$, www.itu. int/rec/R-REC-P/e, 2001-2003.

DMF: www.emf-forschungsprogramm.de/forschung, 2006. 